\title{
Evaluating the Effects of Anti-Viral Therapy on Insulin Resistance and Diabetic Status in Chronic Hepatitis C patients Gamal Ahmed Tawfeek ${ }^{1}$, Abd El-Raoof El-Deib ${ }^{1}$, Fadia Mostafa Attia ${ }^{3}$, Nader El-Nemr ${ }^{2}$, Asmaa Salman Faisal ${ }^{4}$, Ahmed Salman Faisal ${ }^{2}$ \\ ${ }^{1}$ Department of Internal Medicine, Suez Canal University, Ismaillia, 41522, Egypt \\ ${ }^{2}$ Department of Endemic and Infectious Diseases, Suez Canal University, Ismaillia, 41522, Egypt. \\ ${ }^{3}$ Department of Clinical Pathology, Suez Canal University, Ismaillia, 41522, Egypt. ${ }^{4}$ Department of Internal Medicine, Elkantara West Hospital-Ismailia Governorate.
}

Corresponding Author Ahmed Salman Faisal

Mobile:

+ 20966595940065

E mail:

Ahmedf65@yahoo.com

Key words:

Clostridium Difficile, inflammatory bowel disease, ulcerative colitis, Crohn's disease
Background and study aim: Chronically Hepatitis C virus (HCV)-infected patient, develops many complications, as liver cirrhosis, diabetes, and hepatocellular carcinoma (HCC). This study aimed at evaluation of direct acting anti-viral therapy effect on insulin resistance (IR) and glycemic status in $\mathrm{CHC}$ patients with type 2 diabetes mellitus, 3 months after the end of treatment.

Patients and Methods: This prospective work included 72 (better patients) with $\mathrm{CHC}$ and type 2 diabetes. All were enrolled for anti-viral therapy at the center for treatment of viral hepatitis in Ismailia Governorate during the period from September 2015 to September 2016. Data

\section{INTROUUTION}

$\mathrm{HCV}$ infection is a major public health problem, with an estimated worldwide prevalence rate of $2.5 \%$ (about 177.5 million infected adults); which ranges from $1.3 \%$ in the Americas to $2.9 \%$ in Africa [1].The patient infected with hepatitis $\mathrm{C}$ virus, may progress to chronic hepatitis $\mathrm{C}$ $(\mathrm{CHC})$, liver cirrhosis and liver cell failure, and HCC and is associated with high prevalence of type 2 diabetes mellitus [2]. About 10-20\% of chronic hepatitis $\mathrm{C}$ patients progress to liver cirrhosis [3]. Chronic hepatitis $\mathrm{C}$ seems to be responsible for $50 \%$ to $76 \%$ of all liver cancers globally and also for two-third of all cases of liver transplantation [4]. Liver mortality in Egypt comes second after heart diseases, and explains about 40,000 death per year, which constitutes $10 \%$ of total mortality [5]. from patients' candidate for anti-viral therapy was collected at pretreatment and after 12 weeks of therapy. The data including, FBS, IR by Homa IR index and quantitative HCV-RNA.

Results: There was a significant decrease in the mean fasting blood sugar (FBS) had occurred after treatment with anti-HCV therapy ( $\mathrm{p}<0.0001$ ). Also, a significant decrease in the mean of HOMA-IR had occurred after treatment with anti-HCV therapy $(\mathrm{p}<0.0001)$.

Conclusion: A significant improvement in FBS as well as IR was declared after successful HCV treatment.

Worta Health Organization (WO) and Centers for Disease Control and Prevention (CDC) in USA support the new national strategy for control of hepatitis $\mathrm{C}$ which developed by the National Committee for Control of Viral Hepatitis which has been established in 2006 in Egypt [5]. The first strategy for control of $\mathrm{HCV}$ was implemented in 2008-2012. It Included treatment with pegylated interferon (PEG-IFN) in combination with ribavirin (RBV) [6]. The Ministry of Health (MOH) launched the Plan of Action for the Prevention, Care and Treatment of Viral Hepatitis 2014-2018, as well as the new treatment programme using Sofosbuvir [6]. Sofosbuvir approved by the Food and Drug Administration on, 2013, it is a nucleotide analogue polymerase inhibitor for the treatment of chronic $\mathrm{HCV}$ infection as a component of a combination antiviral treatment regimen. Sofosbuvir-based combinations have very high cure rates that exceeds $90 \%$ in some groups [7]. 
IR is a pre-diabetic phase in which the cells of the body don't respond properly to the normal levels of insulin and need a higher level to achieve the normal metabolic pathways. Insulin resistance is observed in about $25 \%$ of $\mathrm{CHC}$ patients [8]. Insulin resistance contributes to NAFLD directly by increasing de novo lipogenesis and indirectly by increasing free fatty acids (FFA) flux to the liver via decreased inhibition of lipolysis. Several data have reported that liver steatosis is a common histological feature of CHC infection especially caused by genotype 4 [9].

\section{Aim of this work}

In the present study we aimed at identifying the effect of direct acting anti-viral therapy on IR and glycemic status in diabetic patients with CHC.

\section{PATIENTS AND METHODS}

\section{Type and site of study}

This prospective study included 72 patients with CHC and type 2 diabetes. All were enrolled for direct acting antiviral (DAA) therapy at the center for treatment of viral hepatitis in Ismailia Governorate during the period from September 2015 to September 2016., according to these inclusion criteria; Patients with type 2 diabetes mellitus and candidates for $\mathrm{HCV}$ treatment according to the guidelines of national committee for control of viral hepatitis, established by the Egyptian MOHP. Exclusion criteria; Patients with concomitant B infection; other endocrinal diseases as hypothyroidism, Cushing syndrome or receiving concomitant medications my cause insulin resistance as corticosteroids, thiazide diuretic was excluded.

During the study all eligible candidates were subjected to:

(1) All patients were subjected to the following at baseline:

(a) Detailed medical history including; Personal data including age, gender, residency and smoking history, duration of present illness if known, associated chronic diseases as hypertension and Diabetes Mellitus, past history of bilharzial exposure or treatment with tartar emetic injections, blood transfusion, surgical operations and dental procedures.(b) Clinical examination including; general examination, chest, cardiac and abdominal examinations.
Anthropometric measures: Weight, height and body mass index (BMI).

(c) Fundus examination: was performed by ophthalmologist for retinal abnormalities.

(d) Electrocardiogram (ECG): was performed for men over 40 years and women over 50 years.

(e) Abdominal ultrasonography: pelvicabdominal ultrasonography evaluation was performed for all patients.

(f) Laboratory investigations including; Fasting blood Glucose, Fasting blood insulin, IR: determined by HOMA-IR index (HOMA-IR $=$ FPI X FPG $/ 22.5$ (e.g. HOMA of $\geq 2$ indicate IR) (3)

ALT, AST, Total and direct S. bilirubin, Complete blood count (CBC), Prothrombin time, and INR, S. albumin, Hepatitis B surface antigen (HBsAg.), Anti-hepatitis C virus antibody (Anti$\mathrm{HCV} \mathrm{Ab}$ ) (ELISA technique). HCV RNA by polymerase chain reaction (PCR) (qualitative and quantitative), alfa feto-protein.

The selected patients were subjected to direct antiviral therapy in the form of Daclatasvir $60 \mathrm{mg}$ plus Sofosbuvir $400 \mathrm{mg}$ once daily for 12 weeks.

3) All patients were followed up to the end of treatment and up to 12 weeks after the end of treatment by the following:

(a) Measurement of quantitative HCV-RNA.

(b) Fasting blood sugar (FBS), 2-hour post prandial blood glucose (2HPPBG), and fasting insulin level. (c, IR: determined by HOMA-IR index and it is calculated by multiplying fasting plasma insulin (FPI) by, fasting plasma glucose (FPG) then dividing by the constant (22.5);

\section{Data management and statistical analysis}

Collected data was processed using IBM Statistical Package of Social Sciences version 20 (SPSS version 20). Median or means \pm standard deviation (SD) was used to describe quantitative data, and frequency (numbers) and percentages to describe qualitative data. Percentage and rates (\%) were used to describe all categorical variables. Comparison between two means was done by unpaired samples ( $t$ ). for comparison of qualitative variables and significant testing of differences, Chi Squares and Fishers Exact tests were used to test significance of difference for qualitative variables. Pre and post treatment of 
FBS, insulin and HOMA-IR were analyzed using paired student $t$ test. Correlation analysis was performed to evaluate the association between IR and other numeric values only as age, FBS, ALT, AST)

\section{RESULTS}

Our study involved 72 patients with mean age of 48.01 years, $54.3 \%$ of the patients were males and $58.3 \%$ were receiving oral hypoglycemic drugs as a treatment for diabetes mellitus (table 1). Following treatment with DAA, there was significant decrease in the mean level of FBS compared to pretreatment mean, $\mathrm{p}<0.0001$. As shown in table 2.

Also, following treatment with DAAs, there was significant decrease in the mean of HOMA-IR compared to pretreatment mean, $\mathrm{p}<0.05$ (table 3) and graph 1.

Meanwhile, the frequency of patients with abnormal HOMA-IR decreased from 39 (54.2\%) before treatment to $30(41.7 \%)$ after treatment. The difference was statistically significant $(p<0.001)$ (table 4). Furthermore, there a significant difference in the means of FBS was found between the cases of normal and abnormal HOMA-IR either before or after anti-HCV treatment, $\mathrm{p}<0.001$ ) as shown in graph 2.

Beside that we found that there was a significant difference in the means values of insulin was found between the cases of normal and abnormal HOMA-IR either before or after anti-HCV treatment, $\mathrm{p}<0.05$ per table 5 .

Table (1): Shows baseline data of the patients in the both study groups.

\begin{tabular}{|l|l|c|c|}
\hline \multicolumn{2}{|c|}{} & \multicolumn{2}{|c|}{$\mathbf{N}=\mathbf{7 2}$ patients } \\
\hline \multirow{2}{*}{ Age } & Mean \pm SD & \multicolumn{2}{|c|}{$48.01 \pm 8.88$} \\
\cline { 2 - 4 } & Range & \multicolumn{2}{|c|}{$30-70$} \\
\hline \multirow{2}{*}{ Gender } & Female & 33 & $45.8 \%$ \\
\cline { 2 - 4 } & Male & 39 & $54.2 \%$ \\
\hline Antidiabetic treatment & OHD & 42 & $58.3 \%$ \\
\cline { 2 - 4 } & Insulin & 30 & $41.7 \%$ \\
\hline
\end{tabular}

This table shows that the mean ages among the studied group was 48.01 years old, $54.3 \%$ of the patients were males and $58.3 \%$ of them were receiving oral hypoglycemic drugs as a treatment for diabetes mellitus.

Table (2): Shows differences in the means of FBS between pre and post anti-HCV treatment among study group.

\begin{tabular}{|l|c|c|c|}
\hline & $\begin{array}{c}\text { Pre -treatment } \\
\text { FBS }\end{array}$ & $\begin{array}{c}\text { Post- treatment } \\
\text { FBS }\end{array}$ & p-value \\
\cline { 1 - 3 } $\begin{array}{l}\text { Mean } \pm \text { SD } \\
\text { Mg/dl }\end{array}$ & $245.49 \pm 93.03$ & $204.35 \pm 76.95$ & \multirow{2}{*}{$<0.001^{*}$} \\
\hline Range & $113.0-447.0$ & $120.0-420.0$ & \\
\hline
\end{tabular}

*: statistically significant difference

This table shows that a significant decrease in the mean of fasting blood sugar had occurred after treatment with anti-HCV therapy, $\mathrm{p}<0.05$. 
Table (3): Shows differences in the means of HOMA-IR between pre and post anti-HCV treatment among study group.

\begin{tabular}{|l|c|c|c|}
\hline & $\begin{array}{c}\text { Pre -treatment } \\
\text { HOMA-IR }\end{array}$ & $\begin{array}{c}\text { Post- treatment } \\
\text { HOMA-IR }\end{array}$ & \multirow{2}{*}{$<0.001^{*}$} \\
\hline Mean \pm SD & $3.27 \pm 3.42$ & $2.59 \pm 2.54$ & \multirow{2}{*}{ Range } \\
\cline { 1 - 3 }
\end{tabular}

*: statistically significant difference

This table shows that a significant decrease in the mean of HOMA-IR had occurred after treatment with anti-HCV therapy, $\mathrm{p}<0.05$.

Table (4): Shows response difference in the HOMA-IR between pre and post anti-HCV treatment among study group.

\begin{tabular}{|l|c|c|c|c|c|}
\hline \multirow{4}{*}{} & \multicolumn{4}{|c|}{$\mathbf{N}=\mathbf{7 2}$ patients } \\
\cline { 2 - 5 } & \multicolumn{2}{|c|}{ Normal HOMA-IR } & \multicolumn{2}{c|}{ Abnormal HOMA-IR } & \multirow{2}{*}{ p-value } \\
\cline { 2 - 5 } & $\mathbf{N}$ & $\mathbf{\%}$ & $\mathbf{N}$ & $\mathbf{\%}$ & \\
\hline Pre-treatment & 33 & $45.8 \%$ & 39 & $54.2 \%$ & \multirow{2}{*}{$<0.001^{*}$} \\
\hline Post-treatment & 42 & $58.3 \%$ & 30 & $41.7 \%$ & \\
\hline
\end{tabular}

*: statistically significant difference

This table shows that a significant increase in the cases of normal HOMA-IR had occurred after treatment with anti-HCV, nine cases with (12.5\% percentage) changed to normal HOMA-IR after treatment, $\mathrm{p}<0.05$.

Table (5): Shows means of insulin among the normal and abnormal HOMA-IR patients before and after treatment.

\begin{tabular}{|l|l|c|c|c|}
\hline \multicolumn{2}{|c|}{} & $\begin{array}{c}\text { Normal } \\
\text { HOMA-IR }\end{array}$ & Abnormal HOMA-IR & p-value \\
\hline Before & Mean \pm SD & $2.33 \pm 0.72$ & $7.06 \pm 4.17$ & $0.0001^{*}$ \\
\hline After & Mean \pm SD & $2.69 \pm 1.20$ & $8.20 \pm 3.92$ & $0.0001^{*}$ \\
\hline
\end{tabular}

*: statistically significant difference

This table shows that a significant difference in the means of insulin was found between the cases of normal and abnormal HOMA-IR either before or after anti-HCV treatment, $\mathrm{p}<0.05$.

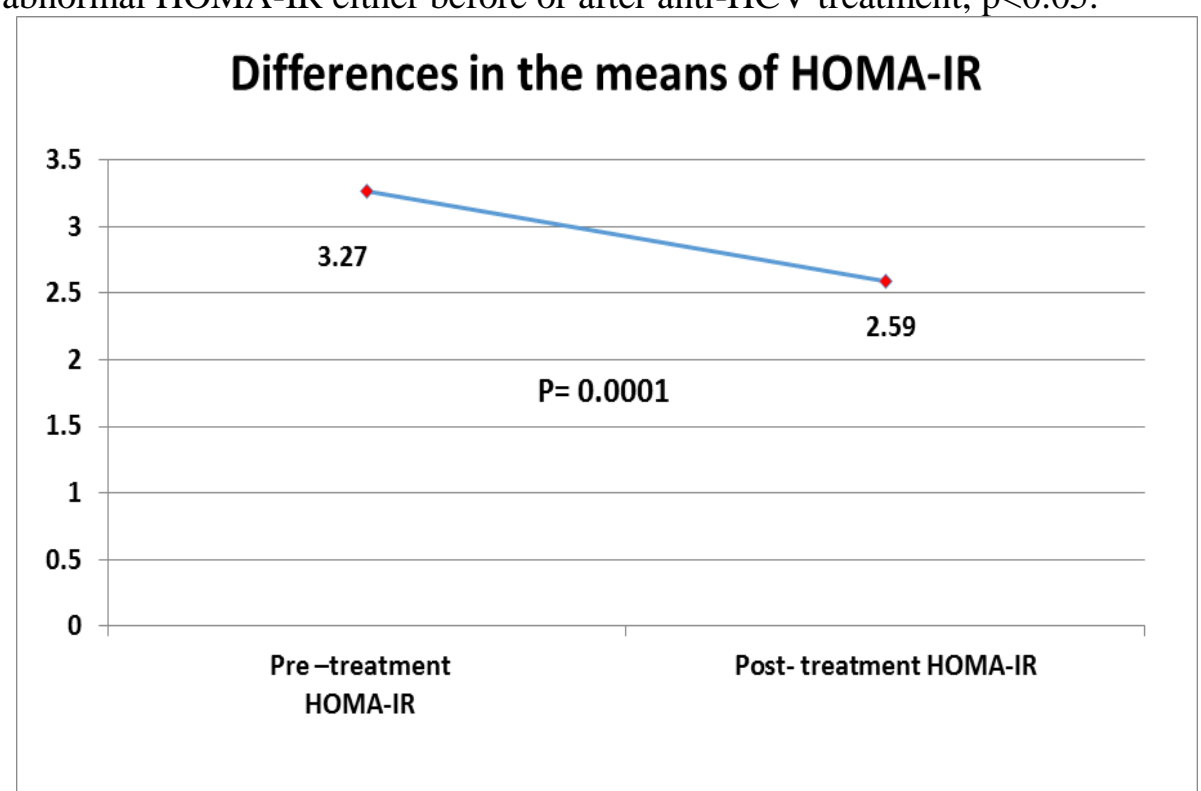

Graph 1: Shows differences in the means of HOMA-IR between pre and post anti-HCV treatment among study group: 


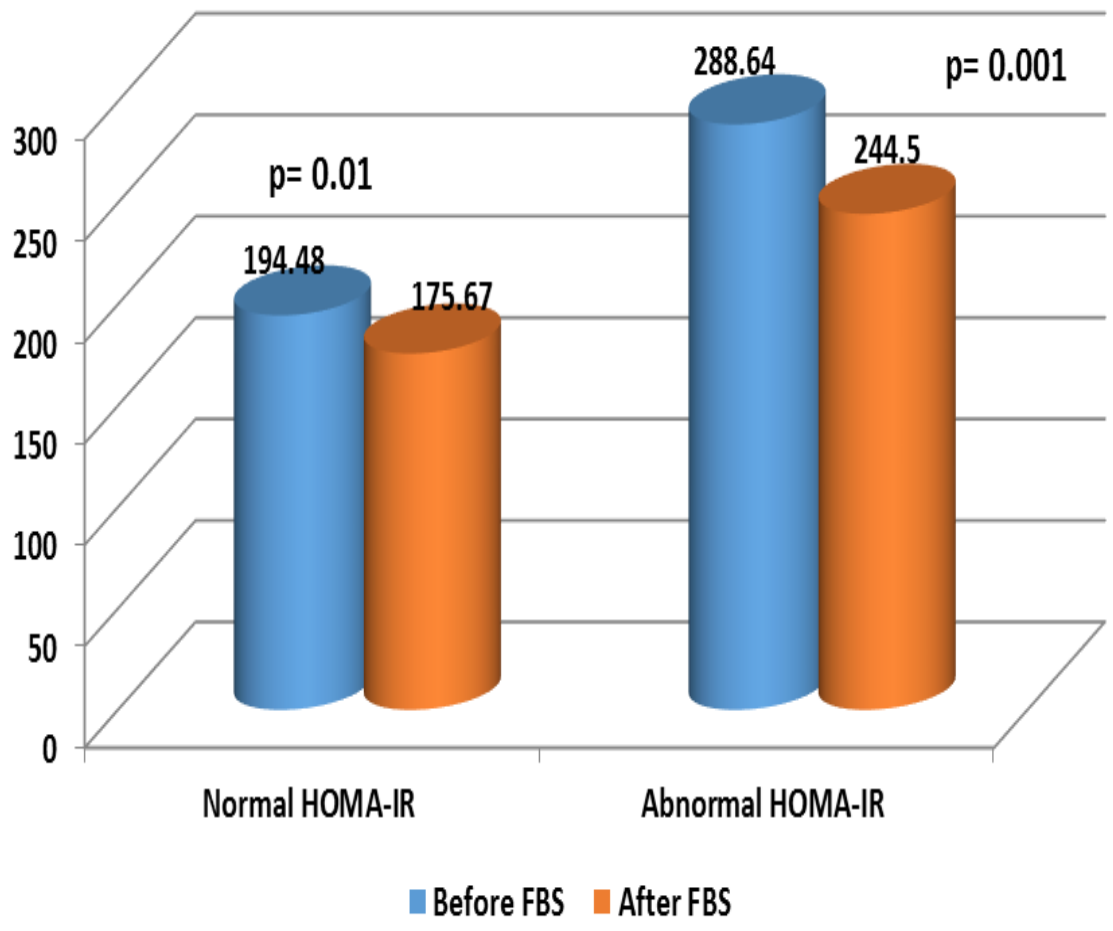

Graph 2: Shows mean FBS among the normal and abnormal HOMA-IR patients before and fter treatment.

\section{DISCUSSION}

Diabetes mellitus type 2 is common in chronic hepatitis $\mathrm{C}$ infection with a higher prevalence than both general population and those with chronic hepatitis B infection, and considered the most common extrahepatic manifestation of chronic hepatitis $\mathrm{C}$ infection [10]. In the present study we aimed at identifying the effect of DAAs therapy on IR and glycemic status in 72 diabetic patients with $\mathrm{CHC}$. The selected patients were subjected to direct antiviral therapy in the form of Daclatasvir 60mg plus Sofosbuvir 400mg once daily for 12 weeks.

Fasting blood sugar showed significant decline after completion of antiviral therapy. Fasting insulin, another marker used to evaluate efficacy of direct acting antiviral therapy, however it revealed no significant change after therapy, on the contrary it showed slight increase after therapy

HOMA-IR which is considered to be most substantial parameter in the study used to evaluate efficacy of antiviral therapy on the diabetic state, compared to its value before therapy, it showed highly significant decrease after therapy.
Our results are consistent with many studies which proved the efficacy of antiviral $\mathrm{HCV}$ therapy in improving insulin resistance [11-13]. These studies also indicated the importance of viral eradication in improving insulin resistance without change in fat mass, level of glucagon, and adipocytokines, consistent with a direct link of the virus with insulin resistance [12]. Moreover, it was shown that insulin resistance by HOMA test, changed in a linear fashion in according to virological response during the antiviral treatment [11].

$\mathrm{HCV}$ infection has an impact on glucose metabolism and may increases insulin resistance, Matsui et al concluded that, HCV replication in vitro, inhibit glucose transporter 2, and decrease glucose uptake by cells [14]. Many studies cleared the relationship between HCV infection and diabetes mellitus type 2 , and proved that patients with chronic HCV infection are more prone to develop T2DM in comparison to a healthy control, or patients with non-viral hepatic diseases [15]. The mechanisms of T2DM in $\mathrm{HCV}$ infected may be due to insulin resistance and failure of glucose cellular re-uptake [16].

IR is presumed to due to, the role of $\mathrm{HCV}$ core protein, and TNF- $\alpha$ which may induce insulin resistance through alterations of receptor's 
signaling of insulin receptors substrate proteins [17].

Recently DAA including sofosbuvir, simeprevir, ledipasvir, and the combination of paritaprevir, ombitasvir achieve SVRs of more than $90 \%$ for most treatment groups [18]. The insulin resistance may affect the response to direct antiviral agents and SVR, as normal glycemic level was shown to be linked to a higher SVR, in a study was done on $\mathrm{HCV}$ infected patient, 24\% of them had diabetes. SVR was $81 \%$ which is lower than the rate reported in previous studies [18]. More studies are needed to confirm the role of DM and insulin resistance in SVR after DAA treatment.

\section{CONCLUSION}

A significant improvement in FBS as well as IR was declared after successful HCV treatment.

Our study has some limitations. First, the small sample size of patients enrolled in the study. Second, we hadn't studied the other different genotypes that could concludes different influences on the link between DAA and IR. Third factor was that our study was observational research and the absence of control group could weaken the data that DAA had an effect on IR in only diabetic patients.

Ethical considerations: The study protocol was approved by the Ethics Committee of Faculty of Medicine, Suez Canal University, the study was explained to eligible patients, and prior consent was to participate in the study.

\section{Funding: No.}

Conflict of interest: No.

\section{REFERENCES}

1. Benova L, Awad SF, Miller FD, Abdu Radad LJ. Estimation of Hepatitis C Virus Infections Resulting From Vertical Transmission in Egypt. Hepatology 2015; 61:834-842.

2. Bruggmann P, Berg T, Ovrehus AL, Øvrehus A L H, Moreno C, Brandão Mello C E, Roudot $\mathrm{F}$, et al. Historical epidemiology of hepatitis $\mathrm{C}$ virus $(\mathrm{HCV})$ in selected countries. $J$ Viral Hepat 2014; 21(Suppl 1):5-33.
3. Rachel H Westbrook 1, Geoffrey Dusheiko, Natural History of Hepatitis C. J Hepatol 2014 Nov; $61(1 \quad$ Suppl): S58-68. doi: 10.1016/j.jhep.2014.07.012. Epub 2014 Nov 3.

4. Iskander D. The Right to Health: a case study on Hepatitis C in Egypt. MS.c. thesis, American University in Cairo. 2013.

5. Cholongitas E, Papatheodoridis G. Sofosbuvir: a novel oral agent for chronic hepatitis C. Ann Gastroenterol 2014; 27(4):331-337.

6. Antonelli A, Ferrari SM, Giuggioli D, Di Domenicantonio A, Ruffilli I, Corrado A, et al. Hepatitis $\mathrm{C}$ virus infection and type 1 and type 2 diabetes mellitus. World J Diabetes. 2014 Oct 15;5(5):586-600.

7. Ezzat WA, Elhosary YA, Abdulla NA, Raslan HM, Saleh MO, Ibrahim MH. Insulin resistance and early virological response in chronic HCV infection. J Gen Eng Biotec 2013; 11:69-73.

8. Knobler H, Schihmanter R, Zifroni A, Fenakel G, Schattner A. Increased risk of type 2 diabetes in noncirrhotic patients with chronic hepatitis $\mathrm{C}$ virus infection. Mayo Clin Proc 2016; 75(4):355-9.

9. White DL, Ratziu V, El-Serag HB. Hepatitis C infection and risk of diabetes: a systematic review and meta-analysis. J Hepatol 2008; 49(5):831-44.

10. Naing J. Ahmed A. Bahk K. Laji. Severe autoimmune insulin resistance-a case report. QJM: An International Journal of Medicine 2012; 107 (12):1019-1021.

11. Mehta SH, Brancati FL, Sulkowski MS, Strathdee SA, Szklo M, Thomas DL. Prevalence of type 2 diabetes mellitus: what is the connection? Ann Intern Med 2000; 133: 592599.

12. Stine JG, Wynter JA, Niccum B, Kelly V, Caldwell SH, Shah NL. Effect of Treatment with Direct Acting Antiviral on Glycemic Control in Patients with Diabetes Mellitus and Chronic Hepatitis C. Annals of Hepatology 2017; 16 (2): 215-220.

13. Doyle MA, Cooper C. "Successful hepatitis C antiviral therapy induces remission of type 2 diabetes: a case report," American Journal of Case Reports 2015; 16: 745-750.

14. Tahrani, L. Bowler, P. Singh, P Coates. "Resolution of diabetes in type 2 diabetic patient treated with IFN- $\alpha$ and ribavirin for hepatitis C," European Journal of Gastroenterology and Hepatology 2006; 18(3): 291-293.

Tawfeek et al., Afro-Egypt J Infect Endem Dis 2020;10(3):287-293

https://aeji.journals.ekb.eg/

http://mis.zu.edu.eg/ajied/home.aspx 
15. Thompson J, Patel K, Chuang WL, Lawitz J, Torres MR, Rustgi K et al. "Viral clearance is associated with improved insulin resistance in genotype 1 chronic hepatitis $\mathrm{C}$ but not genotype 2/3, " Gut 2012; 61(1):128-134.

16. Moucari R, Ripault MP, Martinot-Peignoux M, et al. Insulin resistance and geographical origin: major predictors of liver fibrosis and response to peginterferon and ribavirin in HCV-4. Gut 2009; 58:1662-1669.
17. Pavone P, Tieghi T, d'Ettorre G, Lichtner M, Marocco R, Mezzaroma I et al. Rapid decline of fasting glucose in $\mathrm{HCV}$ diabetic patients treated with direct-acting antiviral agents. Clin Microbiol Infect. 2016 May;22(5):462.e1-3.

18. Premji R., Roopnarinesingh N., N. Qazi, and E. S. Nylen, "New-onset diabetes mellitus with exposure to ledipasvir and sofosbuvir," Journal of Investigative Medicine High Impact Case $J$ Investig Med High Impact Case Rep. 2015 Dec 29;3(4):2324709615623300. 